\title{
Statistical Properties of the Proportional Voting Process
}

\author{
T.M. GRADOWSKI ${ }^{a, *}$ AND R.A. KosińSKI ${ }^{a, b}$ \\ ${ }^{a}$ Faculty of Physics, Warsaw University of Technology \\ Koszykowa 75, 00-662 Warszawa, Poland \\ ${ }^{b}$ Central Institute of Labor Protection - National Research Institute \\ Czerniakowska 16, 00-701 Warszawa, Poland
}

We propose a model of elections based on the Sznajd model of social interactions in the stochastic Ravasz-Barabási hierarchical network. The results of numerical calculations for the population described by this model were compared with statistical analysis of elections' results to the lower house of Parliament held in Poland in the years of 2001, 2005 and 2007. It is shown that the distribution of votes among candidates for the whole country as well as for the district of Warsaw has the log-normal character. The results from Poland are compared with other countries: Brazil and India. For all the cases log-normal distribution of votes for the candidates from each political party is shown. The probability of gaining a number of votes as a function of the number of the candidate on the list is presented.

PACS numbers: 11.25.Hf, 87.23.Ge

\section{Introduction}

Investigations and modelling of social systems have become an important field of many physicists' scientific activity. It appears in different aspects, e.g. cultural diversity of the society, epidemic spreading [1] or opinion formation [2]. An example of this last case is the election process.

Election is the most crucial issue in modern democratic society. It is a complex decision making process, which depends on the involvement of all members of the society, politics, activists, organisations and mass-media. It is the most important and most effective way for society to affect itself.

Investigations concerning the statistical analysis of the elections' results towards the application of the proper models of social interacting may be helpful in understanding a complexity of opinion formation process; they can also be important in prognosis of the results of elections.

${ }^{*}$ corresponding author; e-mail: tomgrad@if.pw.edu.pl 
It has been shown previously that the distribution of votes among the candidates in Brazil follows a power law [3]. Similar research has also been done on the results from few other countries and resembling results were obtained [4-6]. In this paper we show that results of Polish elections have a log-normal distribution. We also try to explain this phenomenon with the proposed simple model of election.

\section{The model of opinion formation}

To simulate the process of voting, we introduce a network model based on well known Sznajd model of personal interactions [7] with the topology of RavaszBarabási (RB) hierarchical network. The RB network has many of the features which are present in real networks, in particular social ones: it has power law degree distribution (scale-free phenomenon), it has short average length of the path (small-world phenomenon), the clustering coefficient is high and depends on the node degree hyperbolically, and the structure is hierarchical. In our model, population of $N$ individuals is represented by the nodes of a $\mathrm{RB}$ stochastic hierarchical network and the time evolution of the opinions in the population are described using the Sznajd model (united we stand, divided we fall), originally proposed for one dimension. Connection of each node with its nearest neighbours means an interpersonal contact ability. Each individual is described by its opinion $S_{i} \in[1, q]$, where $q$ is the number of candidates in the election, so each opinion describes a single vote for a candidate to the parliament. As an initial state $\left\{S_{i}(t=0)\right\}$ opinions of individuals in the population are randomly chosen with equal probability. Evolution of these opinions are simulated numerically by a Monte Carlo procedure. Each Monte Carlo step (MCS) contains $N$ elementary steps. In each elementary step a pair of neighbouring nodes, $i-j$, is randomly selected without repetition. If they share the same opinion $\left(S_{i}=S_{j}\right)$, they can influence their neighbourhood. One individual from their nearest neighbours is randomly selected and its opinion is changed to $S_{i}$. In our simulations we investigate the distribution of votes among the candidates in presence of temperature. The temperature is a measure of noise and gives the effect of an external information impact. In our model the temperature is introduced as a probability, that an individual will not listen to his neighbours in the Sznajd dynamics.

Construction of the RB hierarchical, stochastic network is based on the iterative replication procedure [8]. We start creating a fully connected cluster of five nodes. In each iteration step the existing module is replicated 4 times, and randomly chosen $p^{k}$ fraction (where $k$ is the number of current iteration) of its nodes is connected to one of the nodes from the central module, using preferential attachment rule. If we repeat the procedure $n$ times, we have a module consisting of $5^{n+1}$ nodes.

We performed numerical simulations of the model for the network consisting $N=5^{9}=1953125$ individuals and two different numbers of candidates: $q=200$ and $q=785$. The distributions of votes obtained by the candidates are shown 


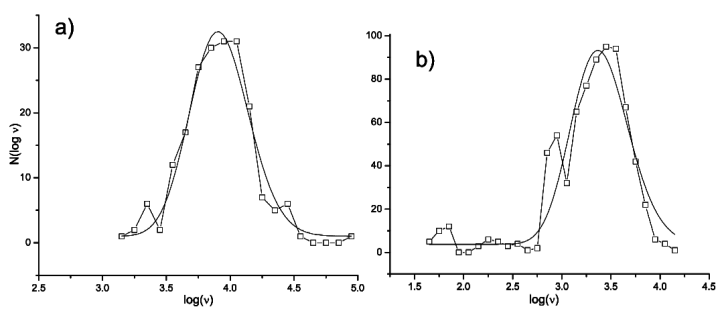

Fig. 1. The histograms of the number of candidates $N(\nu)$ who got a logarithm of fraction $\nu$ of votes - results from the model for $N=5^{9}=1953125$ voters in temperature $T=0.02$ and number of candidates $q=200$ (a) and $q=785$ (b). Mean values are: 9765.6 (a) and 2621.6 (b). Median values are 8328.5 (a) and 2387.1 (b). Solid line represents the log-normal fit.

in Fig. 1. Solid line represents the log-normal fit. Characteristic outcome for a small number of candidates result is not only a small median value, which is obvious, but also hyperbolic right slope. In case of big $q$ values the probability of gaining a very small number of votes is much higher than in case of small number of candidates. This feature causes some fluctuations visible in Fig. 1b for small values of fraction $\nu$. The disturbance of the log-normal fit is also a consequence of this feature.

\section{Results}

Proportional voting system is the most often used electoral formula. In this system representation is chosen proportionally to the number of votes obtained by a party-list.

Particularly, in Polish elections to the Parliament the whole country is divided into 41 districts. Each district has separate party-lists. In each one a number of representatives is chosen and equals 7 up to 19 representatives in the biggest district (460 representatives totally), according to the population of a district.

To obtain the distribution of votes for the whole country we normalize number of votes of each candidate by the total sum of votes from her or his district and we multiply this fraction by the sum of votes from the whole country (just to avoid negative logarithm values). Finally we group the number of candidates $N(\nu)$ which got a logarithm of certain fraction of votes $\nu$, in a histogram.

In Fig. 2 we show the distribution of votes from the whole country, obtained in the following three elections: in 2001, in 2005, and 2007. Solid line represents the log-normal fit computed with the Levenberg-Marquardt algorithm. Voting distributions for single voting district obtained for the biggest district of Warsaw are shown in Fig. 3. The shapes of the distributions for 2005 and 2007 are slightly different. This situation is caused by the differences between the number of candidates in elections, as well as by the much different voter turnout. In the early elections in 2007 twice less candidates were registered for the election than in 2005. 
The voter turnout in 2005 was $40.57 \%$, but in 2007 it was $53.88 \%$. That is why the median and mean values in the year of 2007 are twice higher comparing to the results from 2005. Also we can compare a shape of distribution, in particular the right slope for the results from the 2007 is close to the hyperbolic profile.

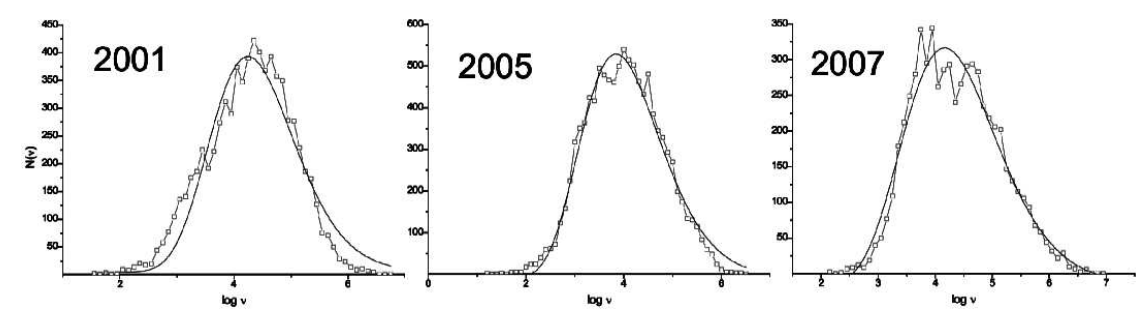

Fig. 2. The histograms of the number of candidates $N(\nu)$ who got a logarithm of fraction $\nu$ of votes in the elections in Poland in 2001, 2005, and 2007. The number of the total votes and candidates were: in $2001-13,017,929$ votes, 7508 candidates; in 2005 - 11,804,676 votes, 10658 candidates; in 2007 - 16,142,202 votes, 6196 candidates. The average/median values were: 1733/515 (2001), 1107/242 (2005), 2605/509 (2007).

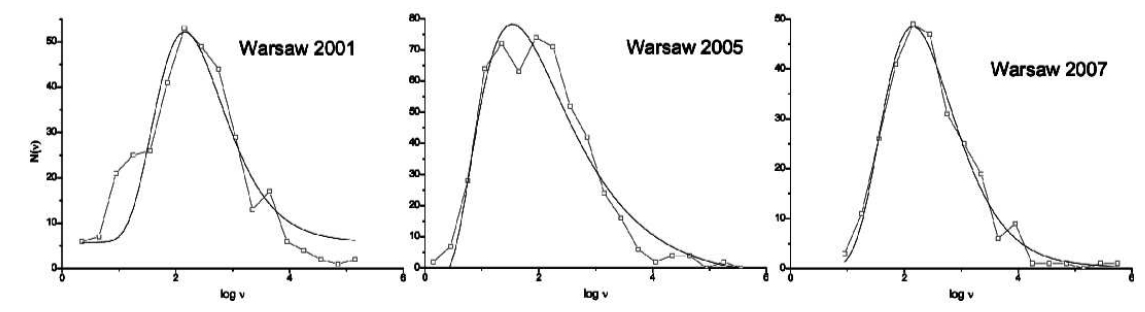

Fig. 3. The histograms of the number of candidates $N(\nu)$ who got a logarithm of fraction $\nu$ of votes in the elections in Warsaw in 2001, 2005, and 2007. The number of the total votes and candidates were: in $2001-734,394$ votes, 346 candidates; in $2005-758,513$ votes, 534 candidates; in $2007-1,145,983$ votes, 272 candidates. The average/median values were: 2122/179 (2001), 1420/78 (2005), 4213/216 (2007).

Let us compare the results of elections in Poland, Brazil [3], and India [4]. The differences in the histograms $N(\nu)$ between Poland, Brazil, and India may be caused by the big differences between number of candidates and the voter turnout. In particular in Brazil the average voter turnout is $83 \%$ due to compulsory voting [9]. In Poland it is $51 \%$. Also the number of candidates per voter in Poland is about 10 times higher than in Brazil and about 40 times higher than in India. So we can expect that with higher voter turnout and much less number of candidates the distribution of votes in Polish elections would be hyperbolic, just like in Brazil.

Similar distributions of the votes gained by candidates from the single list, averaged over all lists from the whole country is shown in Fig. 4. The log-normal distribution is visible as well. 


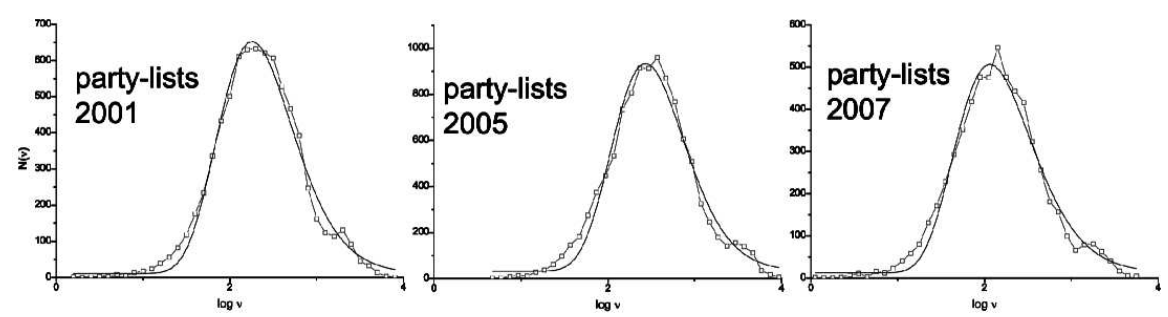

Fig. 4. Distribution of votes on the lists, averaged over all lists in the country.

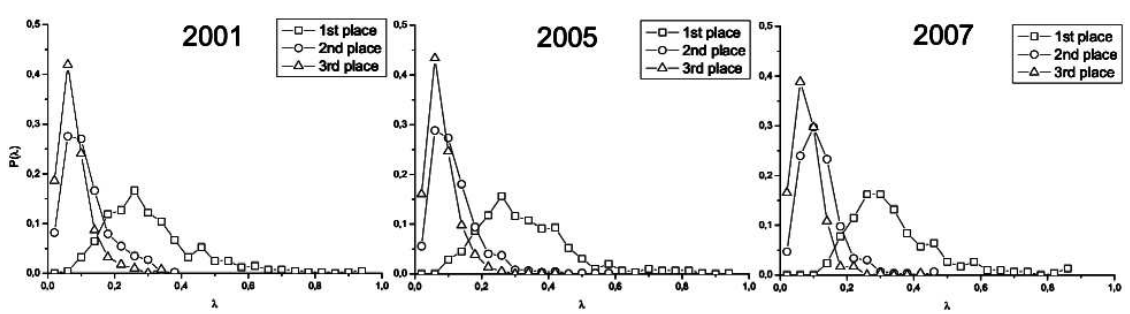

Fig. 5. Probability $P(\lambda)$ that first (squares), second (circles) and third (triangles) candidate on the list will get fraction $\lambda$ of votes. Results from years of 2001, 2005, and 2007 averaged over all lists in all electoral districts.

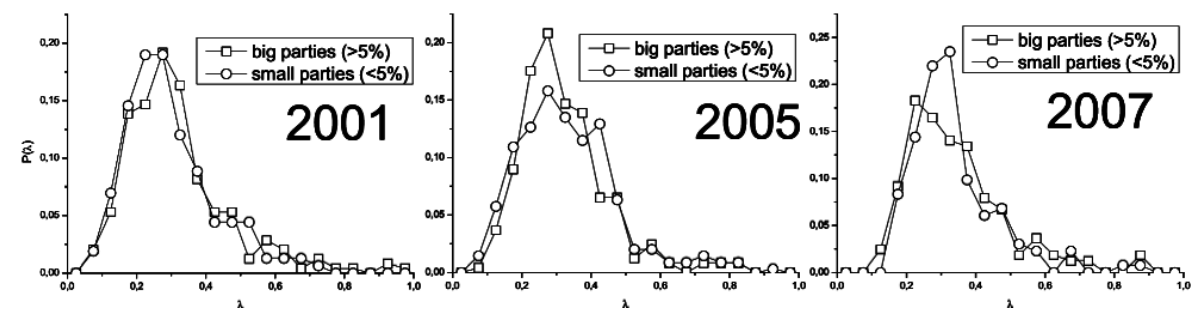

Fig. 6. Probability $P(\lambda)$ that first candidate on the list will get fraction $\lambda$ of votes - comparison of small parties, with overall result below 5\% (circles) and big parties (squares).

In Fig. 5 we show the probability of getting a $\lambda$ fraction of votes from the whole list by first candidate on the list, and for comparison, by second and third candidate on the list. This result shows the phenomenon of the proportional voting: the first candidate on the list is mostly selected. The non-zero value of $P(\lambda)$ even for high values of $\lambda(\lambda>0.5)$ shows that there are some parties, whose result is based on popularity of a single candidate in the district. We also examined this phenomenon separately for small parties (with overall result less than $5 \%$ ) and for big parties (Fig. 6). Results show that this phenomenon occurs as well for small as for big parties and is common for the proportional voting system: voters often vote for the first candidate on the list. We can interpret this behavior as voting for the list (party), not for the candidate. 


\section{Conclusions}

We performed a statistical analysis on the results of proportional elections in Poland and we showed a good agreement of the distribution of votes among the candidates with the log-normal distribution. We also showed that candidates who are first on the party list are getting the most of votes. This may be caused by the tendency of voting for the party, not for the particular candidate. There is no constant difference between small and big parties in this aspect. We proposed a simple voting model, based on Sznajd model of personal interactions and previous attempts. Results from our model follows the log-normal distribution.

\section{References}

[1] R. Pastor-Satorras, A. Vespignani, Phys. Rev. E 63, 066117 (2001).

[2] P.R.A. Campos, V. de Oliveira, F.G. Brady Moreira, Phys. Rev. E 67, 026104 (2003).

[3] R.N.C. Filho, M.P. Almeida, J.S. Andrade, Jr., J.E. Moreira, Phys. Rev. E 60, 1067 (1999).

[4] M.C. Gonzalez, A.O. Sousa, H.J. Herrmann, Int. J. Mod. Phys. C 15, 45 (2004).

[5] A.T. Bernardes, U.M.S. Costa, A.D. Araujo, D. Stauffer, Int. J. Mod. Phys. C 12, 159 (2001)

[6] A.T. Bernardes, D. Stauffer, J. Kertesz, Eur. Phys. J. B 25, 123 (2002).

[7] K. Sznajd-Weron, J. Sznajd, Int. J. Mod. Phys. C 11, 1157 (2000).

[8] E. Ravasz, A.-L. Barabasi, Phys. Rev. E 67, 026112 (2003).

[9] M.N. Franklin, Electoral Participation, Controversies in Voting Behavior, 4th ed., CQ Press, Washington 2001, p. 83. 